\title{
Current patient perspectives of vulvovaginal candidiasis: incidence, symptoms, management and post- treatment outcomes
}

Junko Yano ${ }^{1}$, Jack D. Sobel ${ }^{4}$, Paul Nyirjesy ${ }^{5}$, Ryan Sobel ${ }^{6}$, Valerie L. Williams ${ }^{3}$, Qingzhao Yu $^{2}$, Mairi C. Noverr ${ }^{1,7}$ and Paul L. Fidel $\mathrm{Jr}^{1 *}$ (D)

\begin{abstract}
Background: Vulvovaginal candidiasis (WC) is a common infection affecting women worldwide. Reports of patterns/risk factors/trends for episodic/recurrent WC (RWC) are largely outdated. The purpose of this study was to obtain current patient perspectives of several aspects of WC/RWC.

Methods: Business cards containing on-line survey information were distributed to healthy volunteers and patients seeking standard, elective, or referral gynecologic care in university-affiliated Obstetrics/Gynecology clinics. The internet-based questionnaire was completed by 284 non-pregnant women (78\% Caucasian, 14\% African American, 8\% Asian).

Results: The majority of the participants (78\%) indicated a history of WC with 34\% defined as having RWC. The most common signs/symptoms experienced were itching, burning and redness with similar ranking of symptoms among WC and RWC patients. Among risk factors, antibiotic use ranked highest followed by intercourse, humid weather and use of feminine hygiene products. A high number of respondents noted 'no known cause' (idiopathic episodes) that was surprisingly similar among women with a history of either WC or RWC. WC/RWC episodes reported were primarily physician-diagnosed (73\%) with the remainder mostly reporting self-diagnosis and treating with over-the-counter (OTC) medications. Most physician-diagnosed attacks utilized a combination of pelvic examination and laboratory tests followed by prescribed antifungals. Physician-treated cases achieved a higher level of symptom relief (84\%) compared to those who self-medicated (57\%). The majority of women with RWC (71\%) required continual or long-term antifungal medication as maintenance therapy to control symptoms.
\end{abstract}

Conclusions: Current patient perspectives closely reflect historically documented estimates of WC/RWC prevalence and trends regarding symptomatology, disease management and post-treatment outcomes.

Keywords: Vaginitis, Vulvovaginal candidiasis, WC, RWC, Epidemiology, Incidence rates, Candida albicans, Symptomatology, Risk factors, Disease management

\footnotetext{
* Correspondence: pfidel@lsuhsc.edu

${ }^{1}$ Center of Excellence in Oral and Craniofacial Biology, School of Dentistry,

Louisiana State University Health Sciences Center, New Orleans, LA, USA

Full list of author information is available at the end of the article
}

(c) The Author(s). 2019 Open Access This article is distributed under the terms of the Creative Commons Attribution 4.0 International License (http://creativecommons.org/licenses/by/4.0/) which permits unrestricted use, distribution, and reproduction in any medium, provided you give appropriate credit to the original author(s) and the source, provide a link to the Creative Commons license, and indicate if changes were made. The Creative Commons Public Domain Dedication waiver (http://creativecommons.org/publicdomain/zero/1.0/) applies to the data made available in this article, unless otherwise stated. 


\section{Background}

Vulvovaginal candidiasis (VVC) is a common fungal infection caused by Candida species, predominantly $C$. albicans [1]. Historical reports approximate that $70 \%$ of all women will have at least one episode of VVC during their reproductive years [2]. The pathological hallmark of the disease is an acute inflammatory condition of the vulva and vaginal mucosa induced by and accompanied with overgrowth of Candida organisms that normally exists as a quiescent vaginal commensal [3]. Signs/symptoms of VVC are typically characterized by white clumpy discharge, burning, redness and itching in the vulva and vagina, and dyspareunia [4]. The onset of most VVC cases is believed to be associated with a wide range of predisposing factors or triggering events including the use of antibiotics, increased estrogen levels (e.g. high estrogen oral contraceptives, hormone replacement therapies, pregnancy), uncontrolled diabetes mellitus, sexual activities and tight-fit clothing [2, 5]. In addition, an estimated 8 to $10 \%$ of women are susceptible to recurrent VVC (RVVC), having 4 or more episodes per annum [4]. Multiple recurring infections are often idiopathic without regard to the array of potential risk factors. Unlike most episodic or sporadic VVC, RVVC cases require maintenance regimens with long-term use of antifungals over several months or longer to avoid recurrence $[4,6]$.

Despite the high incidence rates worldwide, epidemiological data supporting the current estimates of VVC or RVVC prevalence rates had been limited, largely historical, and often anecdotal. More recently, however, several global studies have been reported. One reports the worldwide prevalence of RVVC at approximately 138 million women annually, and an additional 372 million over one's lifetime, causing substantial morbidity and economic burden [7]. Another large-scale multi-country internet panel survey indicated a lifetime RVVC prevalence rate of $9 \%$ by age 50 with the vast majority of episodes occurring between 19 to 35 years of age [8], which was remarkably similar to the historical estimates [9]. Despite these global studies on incidence/prevalence rates worldwide, current information/perspectives on VVC/RVVC disease trends is still needed and critical for reporting on aspects of vaginal infections, including diagnostics and therapeutic approaches, understanding host immunity and pathogenesis, and behavioral factors associated with disease etiology. Indeed, a large body of current literature on host defense mechanisms against VVC/RVVC and associated immunopathology [10-14] has relied on long past historical epidemiological citations for VVC/RVVC that may not accurately reflect current disease-associated trends.

The objective of this study was to conduct a contemporary survey of women with a history of VVC and RVVC among general populations and those seeking standard or elective care at several university-affiliated Obstetrics and Gynecology clinics or referral clinics, to determine current trends including diagnostic/management parameters, ranking of disease symptomatology, and post-treatment outcomes, in addition to prevalence rates.

\section{Methods}

\section{Study designs}

The survey included responses by 284 non-pregnant women over a period of February 2016 to May 2018. Business cards containing on-line survey information were distributed to subjects who sought gynecologic care in university-affiliated Obstetrics and Gynecology clinics at the time of visit with a health care provider or via group encounters (meetings, classes, community activities). The university-affiliated $\mathrm{Ob} / \mathrm{Gyn}$ clinics included the following: Detroit Medical Center, Drexel University, Louisiana State University Health Sciences Center (LSUHSC) - New Orleans and Thomas Jefferson University. Eligible subjects (any adult women) participated in the survey on a voluntary basis by accessing a specified URL via internet and answering a questionnaire using Survey Monkey (http://www.surveymonkey.com). Access to the survey web site was open to the general public without password restriction. The questionnaire was approved by the Institutional Review Board at LSUHSC, New Orleans with a waiver of informed consent. The survey was administered anonymously and included no identifiers linking data to individual respondents.

\section{Survey contents and interpretations}

The survey questionnaire included a total of 13 questions regarding i) participant demographics, ii) previous history of VVC, iii) lifetime/annual frequencies of VVC episodes, iv) vulvovaginal signs and symptoms, v) known causes if any, vi) choice of clinical interventions for diagnoses and treatment regimens, and vii) post-treatment outcomes (Table 1). The questions included 2 to 12 answer choices per question. The participants were presented with the questions one at a time on their device of choice and given the option of 'no response'. The exception was the first question in which participants answering 'no history' were led out of the remainder of the survey. For those questions with more than two answer choices, the responses were combined into two categories for analyses. Not applicable (N/A) answers were interpreted as missing values (Additional file 1).

\section{Statistical analyses}

Fisher's exact test was used for a binomial proportion comparing two populations of women choosing one of two responses (i.e. "yes" versus "no") of the question. For 
Table 1 List of questions and responses in the survey questionnaire

\begin{tabular}{|c|c|}
\hline Questions & Response choices \\
\hline \multirow[t]{3}{*}{ Demographics } & Race \\
\hline & Ethnicity \\
\hline & Age \\
\hline \multirow{2}{*}{$\begin{array}{l}\text { Previous history } \\
\text { of WC/RWC }\end{array}$} & Yes \\
\hline & No \\
\hline \multirow{2}{*}{$\begin{array}{l}\text { Frequency } \\
\text { (lifetime) }\end{array}$} & $1-10$ episodes \\
\hline & $>10$ episodes \\
\hline \multirow{2}{*}{$\begin{array}{l}\text { Frequency } \\
\text { (annual) }\end{array}$} & 0-3 episodes \\
\hline & $>3$ episodes \\
\hline Signs/symptoms ${ }^{a}$ & $\begin{array}{l}\text { Itching, burning, cottage cheese-like discharge, } \\
\text { redness in the vaginal area, vaginal pain, } \\
\text { vaginal dryness, vaginal pain, pain during intercourse }\end{array}$ \\
\hline Causes $^{a}$ & $\begin{array}{l}\text { No known cause, oral contraceptives, antibiotics, } \\
\text { hormone replacement therapy, diabetes, humid } \\
\text { weather, pregnancy, after intercourse, after oral } \\
\text { sex, a new sexual partner, feminine hygiene } \\
\text { products, other }\end{array}$ \\
\hline \multirow[t]{6}{*}{ Diagnosis } & $\begin{array}{l}\text { Physician-diagnosed with exam and lab test, treated } \\
\text { with prescription oral or topical medication. }\end{array}$ \\
\hline & $\begin{array}{l}\text { Physician-diagnosed with exam and lab test, treated } \\
\text { with OTC topical medication. }\end{array}$ \\
\hline & $\begin{array}{l}\text { Physician-diagnosed with exam only, treated with } \\
\text { prescription oral or topical medication. }\end{array}$ \\
\hline & $\begin{array}{l}\text { Physician-diagnosed with exam only, treated with } \\
\text { OTC topical medication. }\end{array}$ \\
\hline & $\begin{array}{l}\text { Self-diagnosed and treated with OTC topical } \\
\text { medication. }\end{array}$ \\
\hline & Other. \\
\hline \multirow[t]{4}{*}{ Relief } & Physician-treated, relief \\
\hline & Self-treated, relief \\
\hline & Physician-treated, no relief \\
\hline & Self-treated, no relief \\
\hline \multirow{2}{*}{$\begin{array}{l}\text { Post-treatment } \\
\text { outcome }\end{array}$} & Cured \\
\hline & Recurred/relapse \\
\hline \multirow{4}{*}{$\begin{array}{l}\text { RWC } \\
\text { management }\end{array}$} & Constant antifungal medication, relief \\
\hline & Constant antifungal medication, no relief \\
\hline & As needed antifungal medication, relief \\
\hline & Avoiding known risk factors without medication \\
\hline
\end{tabular}

aRespondents indicated all applicable choices

questions with multiple answer choices, the entire population of the respondents were categorized into two groups based on criteria specified for each survey data set and analyzed for binomial distributions. For proportions of RVVC patient age ranges, Pearson's Chi-squared tests were used to analyze for equal distributions in each age group. All statistical analyses were performed using the SAS version 9.4 software (SAS Institute, Cary, NC).

\section{Results}

Demographic characteristics of the participants

Race and age group distributions of the survey participants were assessed prior to collecting clinical information in regards to VVC/RVVC. Racial data on respondents who completed the survey included Caucasian $(n=202,77.7 \%)$, African American $(n=37,14.2 \%)$ and Asian $(n=21,8.1 \%)$ women (Table 2). No participants of native American or native Hawaiian/Pacific Islander origins were reported. A small proportion of women identified themselves as Hispanic or Latino $(n=20,8.2 \%)$. Approximately one half of the participants were within reproductive age (18-25 years of age, $n=36$, $13.6 \%$; $26-40$ years of age, $n=131,49.4 \%)$, and the rest were within perimenopausal or postmenopausal age (4155 years of age, $n=59,22.3 \%$; 56 or older, $n=39,14.7 \%$ ).

\section{Prevalence of VVC/RVVC}

Prevalence was determined based on the number of individual VVC/RVVC episodes in a respondent's lifetime and, if any, annually as well. A significantly large proportion of the respondents $(n=220,77.5 \%, p<0.0001)$ reported at least one episode of VVC in their lifetime (Table 2, Fig. 1a). Among those with previous episode(s) of infection, a lower number of respondents reported having $>10$ lifetime episodes $(n=89,43.6 \%)$ compared to those that reported fewer than 10 lifetime episodes $(n=115,56.4 \%, p<0.05)$ (Fig. 1a). Likewise, when evaluated based on the number of VVC episodes per year, the distribution of women with $>3$ episodes (i.e. RVVC)

Table 2 Demographics of study participants with a history of WC/RWC

\begin{tabular}{lll}
\hline Race & Caucasian & $77.7 \%(202)^{\mathrm{b}}$ \\
& African American & $14.2 \%(37)$ \\
& Asian & $8.1 \%(21)$ \\
& Native American & $0.0 \%(0)$ \\
& Native Hawaiian/Pacific & $0.0 \%(0)$ \\
& Islander & \\
& Hispanic or Latino & $8.2 \%(20)$ \\
Ethnic category & Not Hispanic or Latino & $91.8 \%(224)$ \\
& $18-25$ & $13.6 \%(36)$ \\
Age & $26-40$ & $49.4 \%(131)$ \\
& $41-55$ & $22.3 \%(59)$ \\
& $>55$ & $14.7 \%(39)$ \\
& No history & $22.5 \%(64)$ \\
Lifetime history & $\geq 1$ & $77.5 \%(220)$ \\
of infection & $\leq 3$ & $65.4 \%(132)$ \\
Annual frequency & $>3$ (RWC) $^{\text {a }}$ & $34.6 \%(70)$ \\
\hline
\end{tabular}

${ }^{a}$ RVVC, recurrent vulvovaginal candidiasis defined as 4 or more acute episodes in a 12-month period

balues in parentheses indicate the number of respondents who selected each response choice 

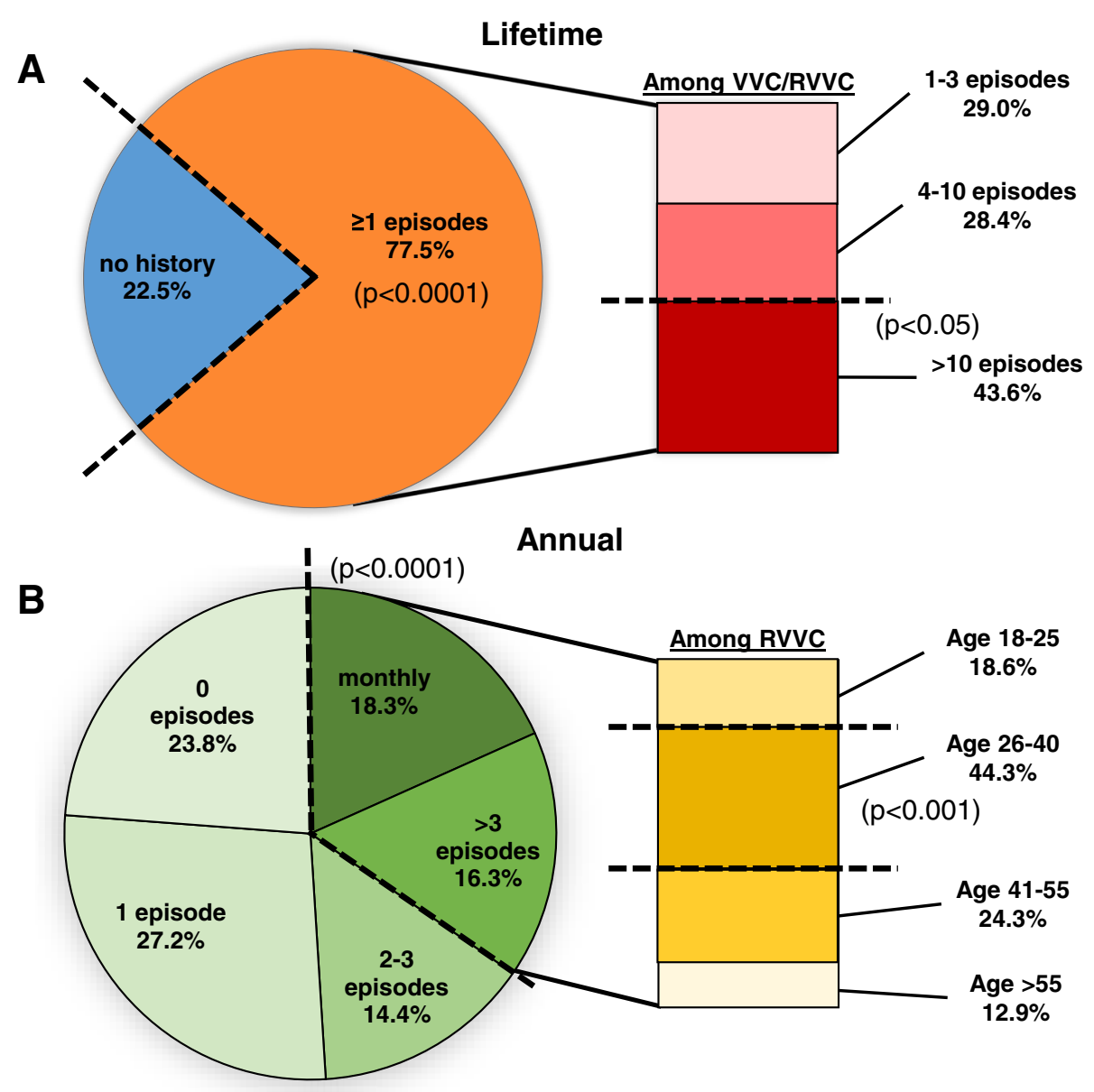

Fig. 1 Prevalence of WC/RWC and distribution of lifetime/annual frequencies of infection. a Lifetime history of WC in participating women $(n=284$, pie chart) was assessed by a self-reported survey. Total WC episodes in respondents with disease history were further stratified by lifetime frequencies ( $n=204$, bar chart). $\mathbf{b}$ Respondents with previous WC episodes were classified by annual frequencies $(n=202$, pie chart), and those with annual frequencies of $>3$ WC episodes were further stratified by age ( $n=70$, bar chart). The percentage in each section indicates the proportion of women among those who reported answers to each parameter. Data were analyzed by Fisher's exact test for binomial proportions comparing two populations of women categorized by the dashed lines. NS, not significant

( $n=70$ women, $34.7 \%$,) were significantly lower compared to those with $\leq 3$ annual episodes $(n=132,65.3 \%$, $p<0.0001$ ) (Table 2, Fig. 1b). Subsequently, the RVVC population of the respondents were further stratified into specific age groups. The proportion of RVVC women in the range of 26 to 40 years of age was significantly higher than other age groups ranging 18 to 25 years, 41 to 55 years or, $>55$ years $(p<0.001)$ (Fig. 1b).

\section{Clinical features of VVC/RVVC}

Information regarding vaginal signs and symptoms experienced during VVC episodes was reported by both women with infrequent history of infection as well as those with a history of recurrence. Overall, the most common clinical characteristics were associated with vaginal inflammation, namely itching (91.2\% of all respondents; VVC-92.4\%; RVVC-90.0\%) followed by burning (68.3\% of all respondents; VVC-62.1\%; RVVC-81.4\%) (Table 3). Additional common features of symptomatic VVC/RVVC episodes included redness (58.1\%), vaginal discharge described as thick, white or cottage cheese-like (55.6\%), pain during intercourse (40.5\%), vaginal pain (38.1\%) and vaginal dryness (29.3\%). Greater than $50 \%$ of the respondents (55.4\%) reported that their VVC episodes had no known cause. These idiopathic attacks were reported by similar proportions of women with a history of VVC (51.2\%) and RVVC (62.9\%). If a cause was reported, antibiotic use was the most common risk factor (37.8\%) followed by intercourse (21.6\%), hormone-induced conditions (pregnancy, use of oral contraceptives, hormone replacement therapy, 13.7\%) and humid weather (11.3\%). There was moderate association of $\mathrm{VVC} /$ RVVC incidence with use of feminine hygiene products $(10.8 \%)$, having a new sexual partner $(8.3 \%)$ or receptive oral sex $(6.9 \%)$. A small number of cases $(<3.0 \%)$ were reported as diabetes-related. 
Table 3 Clinical features of WC symptomatology and risk factors associated with disease

\begin{tabular}{lll}
\hline Signs/symptoms & Itching & $91.2 \%(187)^{\mathrm{a}}$ \\
& Burning & $68.3 \%(140)$ \\
& Redness in the vaginal area & $58.1 \%(119)$ \\
& Cottage cheese like discharge & $55.6 \%(114)$ \\
& Pain during sex & $40.5 \%(83)$ \\
& Vaginal pain & $38.1 \%(78)$ \\
& Vaginal dryness & $29.3 \%(60)$ \\
Causes & No known cause & $55.4 \%(113)$ \\
& Antibiotics & $37.8 \%(77)$ \\
& After intercourse & $21.6 \%(44)$ \\
& Humid weather & $11.3 \%(23)$ \\
& Use of feminine hygiene product & $10.8 \%(22)$ \\
& Or douching & \\
& Having a new sexual partner & $8.3 \%(17)$ \\
& Pregnancy & $7.8 \%(16)$ \\
After oral sex & $6.9 \%(14)$ \\
& Taking oral contraceptives & $5.4 \%(11)$ \\
Diabetes & $2.5 \%(5)$ \\
Hormone replacement therapy & $0.5 \%(1)$ \\
Others ${ }^{b}$ & $17.2 \%(35)$ \\
\hline
\end{tabular}

Values in parentheses indicate the number of respondents who selected each response choice

${ }^{b}$ Including high sugar diet, exercising, stress, before/after menstruation and swimming

\section{Diagnosis and management of VVC/RVVC}

VVC was either self-diagnosed based on vaginal symptoms alone followed by self-medication with over-the-counter (OTC) antifungals, or diagnosed and treated by a physician. Most respondents in this study had infections diagnosed by physicians (72.9\%), with a smaller proportion (27.1\%) opting self-diagnosis without seeking medical care (Fig. 2a) $(p<0.0001)$. Among women reporting physician-diagnosed VVC/RVVC, the clear majority of cases $(71.7 \%)$ were determined by the combination of performing a pelvic examination for signs of vaginitis and conducting laboratory tests involving wet mount microscopy, cultures, or nucleic acid amplification-based detection to confirm the presence of fungal organisms. Of note, several women reported that physician diagnosis was based upon a pelvic examination alone (28.3\%). Following physician diagnosis, most VVC cases involved treatment with oral or topical antifungal medications prescribed by the physician $(66.5 \%)$ with a minor portion instructed by physicians to treat with OTC medications (7.5\%) (Fig. 2b). Women who reported self-diagnosed VVC (24.1\%) tended to OTC antifungal medications for treatment or occasionally experienced spontaneous clearance of symptoms without treatment (1.9\%).

\section{Clinical outcomes following treatment}

Clinical outcomes of women receiving treatment for VVC/RVVC included a spectrum of symptom control or relief to recurrence of symptoms (often rapid). A significantly larger proportion of women who were treated by physicians achieved relief of symptoms (84.4\% vs. $15.6 \%$ non-relief, $p<0.0001$ ) (Table 4 ). Conversely, the relief rate was considerably lower in women with a history of self-diagnosis and self-directed therapy (57.4\% vs. $42.6 \%$ non-relief, $p=0.06)$. Among those who attained symptom relief following treatment with an antifungal, proportions of women who ultimately experienced a repeat episode and those who reported no further episodes were similar (53.2\% recurrence vs. $46.8 \%$ cure, $p=0.20)$. In the RVVC population, $71 \%$ achieved symptom relief by utilizing maintenance antifungal therapy or on an as-needed basis, whereas $19 \%$ failed to achieve relief of symptoms despite multiple regimens of antifungal medication. A small proportion of RVVC women (9.6\%) reported that avoiding triggering factors or events that they recognized as provoking a symptomatic episode was effective management of RVVC.

\section{Discussion}

Despite decades of research and high global prevalence, VVC and RVVC continue to present major health issues in affected women [2, 7]. Until very recently, general epidemiological information has been limited in contemporary literature and consequently, reports of current research in a variety of areas related to VVC/RVVC have referenced predominantly long past historical epidemiological data/observations. Results from the current survey provide a current patient perspective for several aspects of VVC/RVVC that can be used to compare to historical data and provide a contemporary resource for citation.

Data collected from 284 women showed a high proportion with a history of at least one previous episode of VVC (78\%), with the majority of women with 3 or less annual episodes (65\%) and a smaller proportion of those with 4 or more annual episodes (35\%, i.e., RVVC). If one removes the RVVC sub-population, the lifetime prevalence is reduced to $68 \%$. In either case, the reported lifetime history of $\mathrm{VVC}$ in this population of women is remarkably similar to historical reports $(\sim 75 \%)$ [15-18]. Although the proportion of the RVVC group in this population was relatively high, owing to the inclusion of many women seeking specialist gynecologic care, this provided a large sample size of women with a history of VVC/RVVC $(n=204)$ to provide their perspectives. The race distribution (78\% Caucasian, 14\% African American, and $8 \%$ Asian) was reflective of the participant pool and not designed for matching by age or race. 

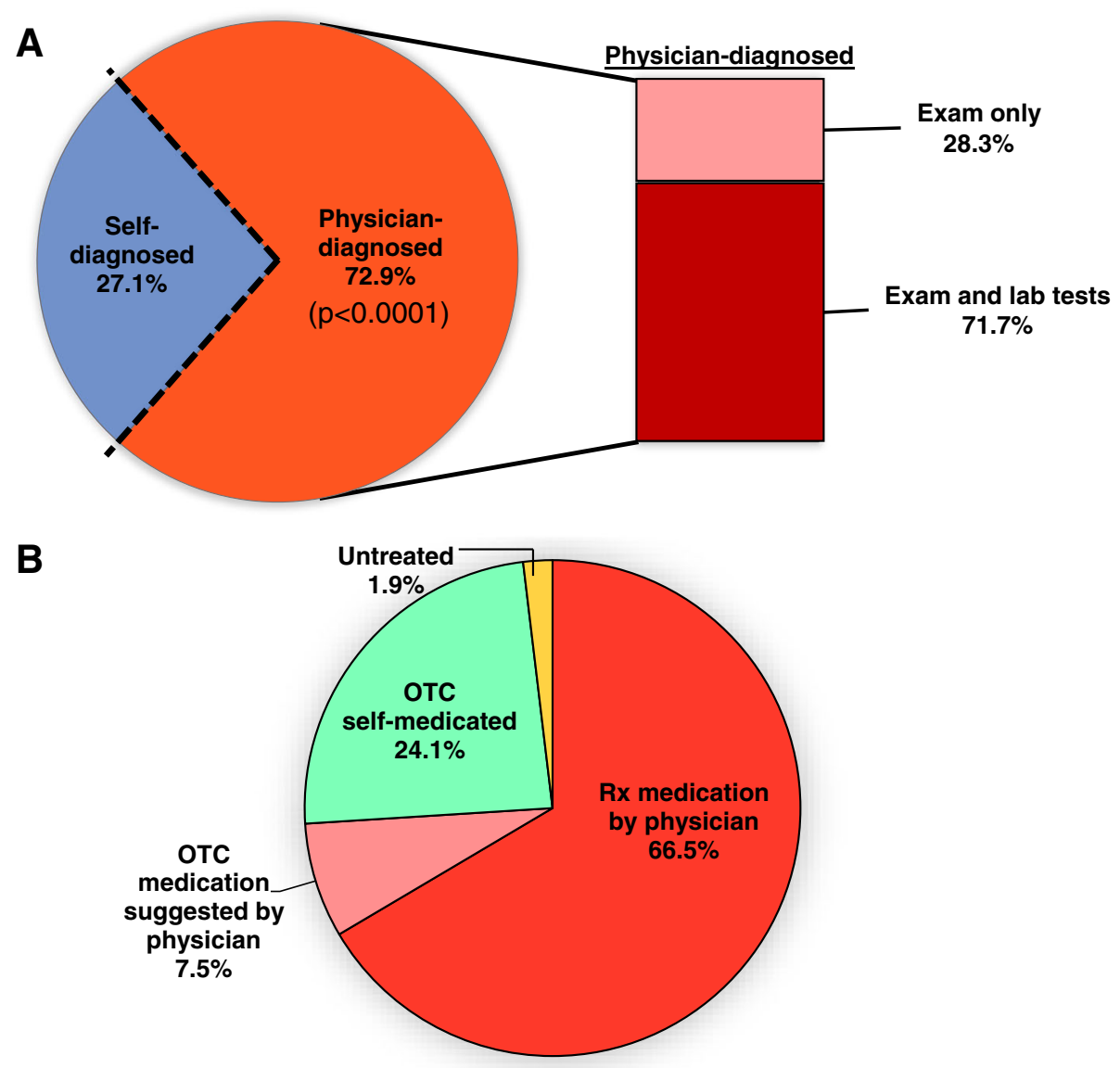

Fig. 2 Methods of disease diagnoses and management in women seeking treatment for vaginitis. a The process of diagnosing vaginitis conditions by participating women ( $n=214$, pie chart) was assessed by a self-reporting survey. Methods of physician-based diagnoses used in respondents seeking medical care were further classified ( $n=152$, bar chart). b The respondents who underwent antifungal treatment $(n=212)$ were categorized based on diagnostic and therapeutic approaches. The percentage in each section indicates the proportion of women among those who reported answers to each parameter. Data (A, pie chart) were analyzed by Fisher's exact test for a binomial proportion comparing two populations of women opting for physician-based diagnosis and self-diagnosis/other (dashed lines). OCT, over-the-counter (non-prescription)

The current survey estimates that approximately $38 \%$ of VVC cases are associated with antibiotic use, similar to previous estimates of 33\% [9]. Interestingly, the high rate of responses (55\%) denoting 'no known cause' for their episodes (idiopathic) was not dominated by RVVC respondents who are characteristically in the idiopathic category relative to cause [4]. Quite the contrary, there was a similar percentage of women with VVC and RVVC noting 'no known cause' ( 52 vs. $63 \%$, respectively). This suggests that while the cause of episodic VVC is often known, there is an equally high proportion of acute VVC cases that are idiopathic similar to RVVC.

As historically reported, signs/symptoms associated with vulvovaginal inflammation (i.e. pruritus, burning, redness) and vaginal discharge are the hallmark manifestations of an acute VVC episode [19], which was also clearly indicated in the current survey. Interestingly, ranking of symptoms reported by those with episodic VVC was comparable to those experienced during
RVVC when analyzed independently. Based on the sufficient numbers of women with VVC and RVVC in the survey, these data have significant power to suggest that the clinical pathology of RVVC is quite similar, if not identical, to acute VVC. It is noteworthy, however, that the frequency of women reporting multiple symptoms was higher in those with RVVC. This may be due simply to the relative frequency of the infection and a higher level of attentiveness to the accompanying signs/ symptoms.

Among the RVVC population, the highest proportion were in the age ranging from 26 to 40 . This is consistent with historical data and likely reflects the reported hormonal and behavioral predisposing factors to infection [20]. The relatively high proportions of RVVC rates in the perimenopausal (41 to 55 years of age) and postmenopausal (56 or older) populations may reflect potential age-related health conditions such as exogenous estrogen use to treat atrophy. Indeed, a study conducted in 
Table 4 Cure rates following treatment

\begin{tabular}{|c|c|c|c|}
\hline & & & $p$-value ${ }^{b}$ \\
\hline \multirow[t]{2}{*}{ Physician-treated } & Relief & $84.4 \%(141)^{\mathrm{a}}$ & $p<0.0001$ \\
\hline & No relief & $15.6 \%(26)$ & \\
\hline \multirow[t]{2}{*}{ Self-medicated } & Relief & $57.4 \%(74)$ & $N S^{c}$ \\
\hline & No relief & $42.6 \%(55)$ & \\
\hline \multirow[t]{2}{*}{ Post-treatment outcome } & Cure & $46.8 \%(94)$ & $N S^{c}$ \\
\hline & Recurrence & $53.2 \%(107)$ & \\
\hline \multirow[t]{3}{*}{$\mathrm{RWC}^{\mathrm{d}}$ maintenance regimens } & Constant/as needed antifungal medication - relief & $71.1 \%(74)$ & $p<0.0001$ \\
\hline & Constant antifungal medication - no relief & $19.2 \%(20)$ & \\
\hline & Avoiding known risk factors without medication & $9.6 \%(10)$ & \\
\hline
\end{tabular}

${ }^{a}$ Values in parentheses indicate the number of respondents who selected each response choice

${ }^{b}$ Data were analyzed by Fisher's exact test for binomial distribution

'NS, not significant

${ }^{\mathrm{d}} \mathrm{RVVC}$, recurrent vulvovaginal candidiasis defined as 4 or more acute episodes in a 12-month period

patients attending a vulvar disease referral clinic reported that nearly $50 \%$ of women diagnosed with $\mathrm{VVC} /$ RVVC were on hormone replacement therapy [21].

Regarding diagnosis and treatment, results showed that a significantly greater proportion of respondents had their episodes physician-diagnosed and treated with prescription antifungal drugs, leading to control/relief of symptoms in $84 \%$ of cases. These observations closely parallel previously reported estimates of 80 to $90 \%$ cure rates (defined by resolution of signs/symptoms and negative mycological tests) by treatment with topical or oral azoles $[22,23]$. Also, consistent with a previous report estimating that only $38 \%$ of cases can be diagnosed correctly by symptomatology alone [19], our survey showed poor relief rate $(57 \%)$ among women who reported they self-diagnosed and self-medicated with OTC drugs. For RVVC, a previous study reported that relapse occurred in $50 \%$ of women who failed to initiate a maintenance regimen [24]. This was reflective in the current survey where $>71 \%$ of women with RVVC reported requiring constant/as needed antifungal therapy to sustain relief. The overall relapse rate noted by the respondents (53\%) is likely reflective of the relatively large RVVC population surveyed.

We acknowledge several limitations to the current study. It is important to note that the VVC diagnosis/ cure data described was solely based on self-reported data as no formal chart screening was performed. As a result, we acknowledge that the open survey format leaves room for inaccuracies that should not be overlooked. For example, poor relief rate among women relying on self-diagnosis may have resulted from self-misdiagnosis. Indeed, signs and symptoms of RVVC are frequently mistaken for many other conditions such as bacterial vaginosis or persistent vulvar vestibulitis, and inadequate diagnosis without clinical examinations often leads to chronic vulvovaginal conditions [7, 25]. But despite potential inaccuracies, these current data are remarkably similar to historical information [19, 22, 23]. The relatively small sample size $(n=284)$ represented another limitation, especially considering the recent internet-based multi-country survey on RVVC prevalence that included 6100 respondents [26]. However, most clinic-based VVC/ RVVC studies consist of sample sizes typical of the present study [3, 27-32]. More importantly, however, is the recognition of selection/accrual bias in the current dataset owing to the fact that the participants were mainly comprised of women seeking care or continued care by a gynecologist, and often those specializing in chronic/recurrent VVC. Indeed, $>34 \%$ of the respondents reported RVVC. This introduced a significant bias in the participant pool that likely resulted in overestimation of the number of physician-diagnosed VVC/RVVC cases although the overall lifetime VVC incidence rate $(\sim 78 \%)$ were comparable to historical estimates $(\sim 75 \%)$ [15-18]. It is assumed that the proportion of self-diagnosed VVC/RVVC cases would be higher in a more randomized population with all respondents devoid of attending established clinics. We recognize that these results may not be fully representative of a broader general population and thus may be another potential limitation. On the other hand, the inclusion of women with higher than average incidence rates of RVVC may actually be considered advantageous as it provided the opportunity to collect more relevant and informative data with respect to symptomatology, diagnosis, treatment and post-treatment outcomes.

In summary, we provide a contemporary patient perspective of several aspects of $\mathrm{VVC}$ and RVVC that while largely confirm historical reports, also include some new insights. Taking into account the overall similarity to historical reports, it bears noting that there has not been any substantial reduction in lifetime or annual prevalence rates of VVC over the past $30+$ years despite a number of new drugs and effective maintenance 
therapy for RVVC. In fact, a study by Denning, et al. predicts an upward trend in RVVC cases by 2030 [7]. This is also despite a plethora of data regarding the pathogenesis of disease and potential immunotherapeutic strategies. Hence, continued research is still needed to impact the incidence of VVC/RVVC. On the positive side, the fact that VVC and RVVC appear to manifest a similar, if not identical, clinical pathology suggests that any diagnostic or immunotherapeutic advances will benefit either condition. Though we recognize the limitations of this study and selection bias of the respondents, these data represent a current set of patient trends in several areas that can serve as a useful resource in future research/publications involving VVC/RVVC.

\section{Conclusions}

A contemporary survey of women with a history of VVC and RVVC provides current trends in prevalence rates, ranking of disease symptomatology, diagnostic/management parameters, and post-treatment outcomes. These current patient perspectives closely reflect previously documented estimates and validate historical reports.

\section{Additional file}

Additional file 1: Participants' responses to the questionnaire. The participants $(n=284)$ were presented with the survey questionnaire items $(\mathrm{Q})$ regarding previous history of WC (Q1), lifetime/annual frequencies of WC episodes (Q2-Q3), vulvovaginal signs and symptoms (Q4), known causes (Q5), choice of clinical interventions for diagnoses and treatment regimens (Q6), post-treatment outcomes (Q7-Q10) and participant demographics (Q11-Q13). A set of 2 to 12 answer choices was included per question. All answer(s) selected by each respondent are indicated (Sheet 1. Raw data). Respondents selecting other as an answer choice in Q5Q6 provided their specified responses as listed in the corresponding spreadsheets (Sheets 2-3. Q5 others and Q6 others). (XLSX 34 kb)

\section{Abbreviations}

OTC: over the counter; RWC: recurrent vulvovaginal candidiasis;

WC: vulvovaginal candidiasis

\section{Acknowledgements}

We thank Ilse Jacobsen at Hans-Knöll Institut for assistance with subject recruitment, and Paul Caballero and Derrick Salvant at LSUHSC for assistance with the survey development (Survey Monkey).

\section{Funding}

This work was supported by the LSUHSC Foundation (PLF), and in part by U54 GM104940 from the National Institute of General Medical Sciences of the National Institutes of Health, which funds the Louisiana Clinical and Translational Science Center. The content is solely the responsibility of the authors and does not necessarily represent the official views of the funders.

\section{Availability of data and materials}

All data generated or analyzed during this study are included in this published article and a supplemental data file.

\section{Authors' contributions}

PLF conceived and designed the study. JDS, PN, RS and VLW distributed survey information in the patient recruitment sites. QY performed statistical analyses. JY and PLF interpreted the data. JY and PLF wrote the manuscript.
JDS, PN, MCN reviewed and edited the manuscript. All authors read and approved the final manuscript.

\section{Ethics approval and consent to participate}

This study was approved by the Institutional Review Board at Louisiana State University Health Sciences Center, New Orleans with Waiver of informed consent.

Consent for publication

Not applicable.

\section{Competing interests}

The authors declare that they have no competing interests.

\section{Publisher's Note}

Springer Nature remains neutral with regard to jurisdictional claims in published maps and institutional affiliations.

\section{Author details}

${ }^{1}$ Center of Excellence in Oral and Craniofacial Biology, School of Dentistry, Louisiana State University Health Sciences Center, New Orleans, LA, USA. ${ }^{2}$ Biostatistics Program, School of Public Health, Louisiana State University Health Sciences Center, New Orleans, LA, USA. ${ }^{3}$ Department of Obstetrics and Gynecology, School of Medicine, Louisiana State University Health Sciences Center, New Orleans, LA, USA. ${ }^{4}$ Department of Internal Medicine, Wayne State University, School of Medicine, Detroit, MI, USA. ${ }^{5}$ Department of Obstetrics and Gynecology, Drexel University College of Medicine, Philadelphia, PA, USA. ${ }^{6}$ Thomas Jefferson University Hospital, Sydney Kimmel Medical College, Philadelphia, PA, USA. ${ }^{7}$ Present Address: Department of Microbiology and Immunology, School of Medicine, Tulane University, 1430 Tulane Ave, New Orleans, LA, USA

Received: 21 June 2018 Accepted: 22 March 2019

Published online: 29 March 2019

References

1. Sobel JD. Epidemiology and pathogenesis of recurrent vulvovaginal candidiasis. Am J Obstet Gynecol. 1985;152(7 Pt 2):924-35.

2. Sobel JD, Faro S, Force RW, Foxman B, Ledger WJ, Nyirjesy PR, et al. Vulvovaginal candidiasis: epidemiologic, diagnostic, and therapeutic considerations. Am J Obstet Gynecol. 1998;178(2):203-11.

3. Fidel PL Jr, Barousse M, Espinosa T, Ficarra M, Sturtevant J, Martin DH, et al. An intravaginal live Candida challenge in humans leads to new hypotheses for the immunopathogenesis of vulvovaginal candidiasis. Infect Immun. 2004;72:2939-46.

4. Sobel JD. Pathogenesis and treatment of recurrent vulvovaginal candidiasis. Clin Infect Dis. 1992;14(Suppl 1):S148-53.

5. Sobel JD. Vaginitis. N Engl J Med. 1997;337(26):1896-903.

6. Achkar JM, Fries BC. Candida infections of the genitourinary tract. Clin Microbiol Rev. 2010;23(2):253-73.

7. Denning DW, Kneale M, Sobel JD, Rautemaa-Richardson R. Global burden of recurrent vulvovaginal candidiasis: a systematic review. Lancet Infect Dis. 2018;18(11):e339-e47.

8. Blostein F, Levin-Sparenberg E, Wagner J, Foxman B. Recurrent vulvovaginal candidiasis. Ann Epidemiol. 2017;27(9):575-82.

9. Sobel JD. Vulvovaginal candidosis. Lancet. 2007;369(9577):1961-71.

10. Fidel PL Jr. History and new insights into host defense against vaginal candidiasis. Trends Microbiol. 2004;12(5):220-7.

11. Peters BM, Yano J, Noverr MC, Fidel PL Jr. Candida vaginitis: when opportunism knocks, the host responds. PLoS Pathog. 2014;10(4):e1003965.

12. Yano J, Noverr MC, Fidel PL Jr. Cytokines in the host response to Candida vaginitis: identifying a role for non-classical immune mediators, S100 alarmins. Cytokine. 2012;58(1):118-28.

13. Cassone A. Vulvovaginal Candida albicans infections: pathogenesis, immunity and vaccine prospects. BJOG. 2015;122(6):785-94.

14. Yano J, Noverr MC, Fidel PL Jr. Vaginal heparan sulfate linked to neutrophil dysfunction in the acute inflammatory response associated with experimental vulvovaginal candidiasis. mBio. 2017:8(2):e00211-7.

15. Hurley R, De Louvois J. Candida vaginitis. Postgrad Med J. 1979;55(647):645-7. 
16. Geiger AM, Foxman B, Gillespie BW. The epidemiology of vulvovaginal candidiasis among university students. Am J Public Health. 1995;85(8 Pt 1):1146-8.

17. Berg AO, Heidrich FE, Finn SD, Bergman JJ, Wood RW, Stamm WE, et al. Establishing the cause of genitourinary symptoms in women in a family practice. Comparison of clinical examination and comprehensive microbiology. JAMA. 1984;251(5):620-5.

18. Horowitz BJ, Edelstein SW, Lippman L. Sexual transmission of Candida. Obstet Gynecol. 1987;69(6):883-6.

19. Anderson MR, Klink K, Cohrssen A. Evaluation of vaginal complaints. JAMA. 2004;291(11):1368-79.

20. Foxman B, Marsh JV, Gillespie B, Sobel JD. Frequency and response to vaginal symptoms among white and African American women: results of a random digit dialing survey. J Women's Health. 1998;7(9):1167-74.

21. Fischer G, Bradford J. Vulvovaginal candidiasis in postmenopausal women: the role of hormone replacement therapy. J Low Genit Tract Dis. 2011;15(4):263-7.

22. Sobel JD, Kapernick PS, Zervos M, Reed BD, Hooton T, Soper D, et al. Treatment of complicated Candida vaginitis: comparison of single and sequential doses of fluconazole. Am J Obstet Gynecol. 2001;185(2):363-9.

23. Reef SE, Levine WC, McNeil MM, Fisher-Hoch S, Holmberg SD, Duerr A, et al. Treatment options for vulvovaginal candidiasis, 1993. Clin Infect Dis. 1995; 20(Suppl 1):S80-90.

24. Sobel JD, Wiesenfeld HC, Martens M, Danna P, Hooton TM, Rompalo A, et al. Maintenance fluconazole therapy for recurrent vulvovaginal candidiasis. N Engl J Med. 2004;351(9):876-83.

25. Farmer MA, Taylor AM, Bailey AL, Tuttle AH, Maclntyre LC, Milagrosa ZE, et al. Repeated vulvovaginal fungal infections cause persistent pain in a mouse model of vulvodynia. Sci Transl Med. 2011;3(101):101ra91.

26. Foxman B, Muraglia R, Dietz JP, Sobel JD, Wagner J. Prevalence of recurrent vulvovaginal candidiasis in 5 European countries and the United States: results from an internet panel survey. J Low Genit Tract Dis. 2013:17(3):340-5.

27. Geiger AM, Foxman B. Risk factors for vulvovaginal candidiasis: a casecontrol study among university students. Epidemiology. 1996;7(2):182-7.

28. de Leon EM, Jacober SJ, Sobel JD, Foxman B. Prevalence and risk factors for vaginal Candida colonization in women with type 1 and type 2 diabetes. BMC Infect Dis. 2002;2:1.

29. Drell T, Lillsaar T, Tummeleht L, Simm J, Aaspollu A, Vain E, et al. Characterization of the vaginal micro- and mycobiome in asymptomatic reproductive-age Estonian women. PLoS One. 2013;8(1):e54379.

30. Foxman B, Barlow R, D'Arcy H, Gillespie B, Sobel JD. Candida vaginitis: selfreported incidence and associated costs. Sex Transm Dis. 2000;27(4):230-5.

31. Geiger AM, Foxman B, Sobel JD. Chronic vulvovaginal candidiasis: characteristics of women with Candida albicans, C glabrata and no candida. Genitourin Med. 1995;71(5):304-7.

32. Mintz JD, Martens MG. Prevalence of non-albicans Candida infections in women with recurrent vulvovaginal symptomatology. Adv Infect Dis. 2013;3(4):5

Ready to submit your research? Choose BMC and benefit from:

- fast, convenient online submission

- thorough peer review by experienced researchers in your field

- rapid publication on acceptance

- support for research data, including large and complex data types

- gold Open Access which fosters wider collaboration and increased citations

- maximum visibility for your research: over $100 \mathrm{M}$ website views per year

At $\mathrm{BMC}$, research is always in progress.

Learn more biomedcentral.com/submissions 